\title{
Fault Diagnosis of a Wind Turbine Benchmark via Identified Fuzzy Models
}

\author{
Silvio Simani, Senior Member, IEEE, Saverio Farsoni, and Paolo Castaldi
}

\begin{abstract}
In order to improve the availability of wind turbines and to avoid catastrophic consequences, the detection of faults in their earlier occurrence is fundamental. The paper proposes the development of a fault diagnosis scheme relying on identified fuzzy models. The fuzzy theory is exploited since it allows to approximate uncertain models and manage noisy data. These fuzzy models, in the form of Takagi-Sugeno prototypes, represent the residual generators used for fault detection and isolation. A wind turbine benchmark is used to validate the achieved performances of the designed fault detection and isolation scheme. Finally, extensive comparisons with different fault diagnosis methods highlight the features of the suggested solution.
\end{abstract}

Index Terms-Data-driven approach, fuzzy modelling and identification, fault detection and isolation, availability and reliability, wind turbine benchmark.

\section{INTRODUCTION}

$\mathbf{I}^{\mathrm{s}}$ $\mathrm{N}$ general, wind turbines in the megawatt size are expensive, and hence their availability and reliability must be high in order to maximise the energy production. Operation and Maintenance $(\mathrm{O} \& \mathrm{M})$ services have to be minimised, since they represent one of the main factors of the energy cost. The capital cost, as well as the wind turbine foundation and installation determine the basic term in the cost of the produced energy, which constitute the energy 'fixed cost'. The O \& M represent a 'variable cost' that can increase the energy cost up to about the $30 \%$. These features motivate the introduction of suitable Fault Detection and Isolation (FDI) systems. The related literature proposes FDI schemes for wind turbines that sometimes are too conservative. For example, turbines are simply turned off after incipient faults to wait for maintenance service. Therefore, effective FDI strategies are needed for improving wind turbine working conditions, even though it might lead to limited power production in case of faults.

In the last years, many works have been proposed on wind turbine FDI, and the most relevant are described e.g. in [1], [2], [3], [4], [5], [6].

Benchmarks are useful for the validation of the robustness and the reliability of fault diagnosis schemes, as proposed e.g. in [7]. This benchmark, which will be considered in the present work, represents a realistic three-bladed horizontal variable

Manuscript received May 1, 2014; revised August 1, 2014; accepted August 31, 2014

Copyright (c) 2014 IEEE. Personal use of this material is permitted. However, permission to use this material for any other purposes must be obtained from the IEEE by sending a request to pubs-permissions@ieee.org.

S. Simani and S. Farsoni are with the Department of Engineering, University of Ferrara, Ferrara, FE, 44122 Italy. E-mail: silvio.simani@unife.it.

P. Castaldi is with the Electric and Informatic Department, University of Bologna, Bologna, Italy.

S. Simani is the corresponding author. speed wind turbine with full scale converter coupling. Several FDI strategies were proposed in the recent literature and compared on this benchmark, as addressed in [7]. Moreover, only the most effective solutions are considered in this paper for comparison purpose, and for highlighting the features of the proposed approach. On the other hand, with respect to the FDI solutions compared in [7], this paper considers a few more FDI strategies that are applied to the benchmark, and described in [8], but for fault tolerant control purpose.

The contribution of this paper consists of the development of a data-driven FDI strategy, which is applied to the same benchmark example. To this aim, the main features of the presented research are highlighted in the following. First, this work proposes a method relying on fuzzy descriptions, thus obviating the derivation of purely nonlinear mathematical models. In some cases, a model-free approach based on dynamic system identification methodologies can be more effective in terms of model complexity and time to achieve it, with respect to classical modelling strategies based on physical laws. This motivation is valid, for example, when the modelling difficulty originates from multiple coupling in system parameters, which are common in large utility-scale wind turbine. Of course, note that, in general, both control and fault diagnosis strategy designs can depends on the system under consideration, as addressed e.g. in [9], [10]. In some cases, wind turbine installations can require accurate modelling and control-oriented engineering models have to be determined from the physical laws ruling the dynamic process under investigation. Secondly, residual generators in the form of fuzzy models are considered, instead of purely nonlinear observer or filters. Third, by exploiting a failure mode and effect analysis, both the system identification procedure and fault isolation task are simplified, thus obviating the need of unknown input or disturbance decoupling designs, as addressed e.g. in [9]. As described in the following, in fact, the paper makes use of Takagi-Sugeno (TS) models [11], whose parameters are obtained via the identification procedure proposed in [12]. This scheme showed remarkable approximation features, also in practical applications, as addressed by the same authors in [13].

Finally, the paper has the following structure. Section II provides a brief overview of the wind turbine benchmark and the considered fault cases. Section III recalls the strategy exploited for obtaining the fuzzy models, which are used as residual generators for the development of the FDI solution. The complete FDI methodology is presented in Section IV. The achieved results that are summarised in Section $\mathrm{V}$ show the performances of the fault diagnosis scheme. Section VI ends the paper by highlighting the main achievements of the 
work, and providing suggestions for further studies.

\section{Wind TURBINE BENCHMARK DESCRIPTION}

The three-bladed horizontal axis wind turbine considered in this paper is the benchmark described in [7]. The rotational speed and the generated power are controlled by means of two controlled inputs: the converter torque $\tau_{g}(t)$ and the pitch angle $\beta_{r}(t)$ of the turbine blades. Several measurements are acquired from the wind turbine system. In particular, $\omega_{r}(t)$ is the rotor speed and $\omega_{g}(t)$ is the generator speed. $\tau_{g}(t)$ represents the torque of the generator controlled by the converter, with respect to the torque reference, $\tau_{r}(t)$. The wind turbine nonlinear aerodynamic model and the noise affecting the considered wind turbine measurements motivate the approach suggested in Section III.

The drive-train of the wind turbine system is modelled by a simple two-mass description, as shown in [7]. The hydraulic pitch system is described as a second order closed-loop transfer function [7]. The converter dynamics are modelled by a first-order transfer function, which provides the power $P_{g}(t)$ from the generator. Finally, the measurement noise signals are modelled as Gaussian stochastic processes with mean and standard deviation values representing the actual measurement sensor accuracy [7].

With these assumptions, the continuous-time description of the wind turbine system has the form (1):

$$
\left\{\begin{array}{l}
\dot{x}_{c}(t)=f_{c}\left(x_{c}(t), u(t)\right) \\
y(t)=x_{c}(t)
\end{array}\right.
$$

where the vectors $u(t)=$ $\left[\beta_{1 m_{i}}(t), \beta_{2 m_{i}}(t), \beta_{3 m_{i}}(t), \tau_{g}(t)\right]^{T}$ and $y(t)=x_{c}(t)=$ $\left[P_{g m}(t), \omega_{g m_{i}}(t), \omega_{r m_{i}}(t)\right]^{T}$ represent the control inputs and the monitored output measurements, respectively. Their components are measured by the $i$-th redundant sensor, with $i=1,2 . f_{c}(\cdot)$ represents continuous-time nonlinear dynamic function describing the behaviour of the wind turbine system. These measurements are sampled for obtaining a number of $N$ input-output data, $u(k)$ and $y(k)$, with $k=1,2, \ldots N$. With reference to the redundant signals, $\omega_{g m_{i}}$ represents the $i$-th generator speed measurement, $\omega_{g m_{i}}$ the $i$-th rotor speed measurement, $P_{g m}(t)$ is the generator power measurement, and $\beta_{j m_{i}}(t)$ the $i$-th pitch measurement of the $j$-th blade. Finally, the model parameters and the map $C_{p}(\beta, \lambda)$ are suitably selected to represent a realistic wind turbine installation [7].

\section{A. Simulated Fault Conditions}

The wind turbine benchmark simulates a number of realistic malfunctions, which are summarised in Table I, and described in more detail in [7].

The relations between the fault cases of Table I and the measurements acquired from the wind turbine system are described in Table II, since this analysis enhances both the design of the fault isolation scheme and the identification approach, recalled in Section III. In particular, Table I highlights that a fault affects mainly only one of the measured inputs and
TABLE I

THE BENCHMARK FAULT SCENARIO.

\begin{tabular}{|c|c|}
\hline Fault & Description \\
\hline 1 & Fixed value of the pitch 1 position sensor 1 \\
\hline 2 & Scaling error of the pitch 2 position sensor 2 \\
\hline 3 & Fixed value of the pitch 3 position sensor 1 \\
\hline 4 & Fixed value of the rotor speed sensor 1 \\
\hline 5 & $\begin{array}{l}\text { Scaling error of the rotor speed sensor } 2 \& \text { the } \\
\text { generator speed sensor } 2\end{array}$ \\
\hline 6 & $\begin{array}{l}\text { Pitch system changed response for the pitch } \\
\text { actuator } 2 \text { due to air content in oil }\end{array}$ \\
\hline 7 & $\begin{array}{l}\text { Pitch system changed response for the pitch } \\
\text { actuator } 3 \text { due to low pressure }\end{array}$ \\
\hline 8 & Offset in the converter torque control \\
\hline 9 & Changed dynamics of the drive train \\
\hline
\end{tabular}

outputs. Moreover, the mismatch between each fault-free and faulty measurement is measured by the Relative Mean Squared Error (RMSE), computed for the different fault cases of Table I.

TABLE II

WIND TURBINE FMEA RESULTS.

\begin{tabular}{c|ccccc}
\hline Measurement & $\beta_{1 m_{1}}(t)$ & $\beta_{2 m_{2}}(t)$ & $\beta_{3 m_{1}}(t)$ & $\omega_{r m_{1}}$ & $\omega_{r m_{2}}$ \\
\hline Fault & 1 & 2 & 3 & 4 & 5 \\
\hline RMSE & 11.29 & 0.98 & 2.48 & 1.44 & 1.45 \\
\hline \hline Measurement & $\beta_{2 m_{1}}(t)$ & $\beta_{3 m_{2}}(t)$ & $P_{g m}(t)$ & $\omega_{g m_{1}}(t)$ & \\
\hline Fault & 6 & 7 & 8 & 9 & \\
\hline RMSE & 0.80 & 0.73 & 0.84 & 0.77 & \\
\hline
\end{tabular}

Note that in Table II the variable $\beta_{i m_{j}}(t)$ indicates the $i-$ th blade pitch $(i=1,2,3)$ measured by the $j$-th redundant sensor $j=1,2$. In the same way, the rotor speed is measured by 2 redundant sensors $\omega_{r m_{j}}(t)$, with $j=1,2 . P_{g m}$ is provided by one sensor only.

It is worth noting that the results summarised in Table II were achieved by performing the so-called Failure Mode \& Effect Analysis (FMEA). Table II is thus obtained by selecting the most sensitive measurements with respect to the simulated fault conditions, represented by the the RMSE values between the fault-free and faulty measured signals.

This methodology is developed for the considered fault scenario, and different measurements could be required for different fault situations. Moreover, a model-free approach to FDI could be based on a general wind turbine model identified in fault-free conditions. The simple discrepancy between the signals from the identified fuzzy model and the actual measurements would provide the fault detection and isolation. On the other hand, if a model-based approach were used, the fault distribution matrices should be known, as described e.g. in [9]. It is also worth observing that the methodology proposed in this article consists of a scheme aiming at detecting and isolating faults in their earlier occurrence, thus allowing lower $\mathrm{O} \& \mathrm{M}$ costs and actions, which usually do not require fault identification. However, once the fault is isolated, it is possible to perform the fault reconstruction, by using an approach similar to the one presented e.g. in [14]. 


\section{FuZZY Modelling AND IDENTIFICATION}

This section recalls the identification method that is exploited for the development of the proposed FDI strategy addressed in Section IV.

In the considered TS model, the rule consequents are crisp functions of the inputs:

$$
R_{i}: \text { IF } x \text { is } A_{i} \text { THEN } y_{i}=f_{i}(x)
$$

with $i=1,2, \ldots, K$. The vector $x$ represents the input (antecedent) variable, whilst $y_{i}$ is the output (consequent) variable. $R_{i}$ denotes the $i$-th rule, and $K$ is the number of rules (or clusters). $A_{i}$ is the antecedent fuzzy set of the $i$-th rule, defined by a (multivariate) membership function [11].

The consequent $f_{i}$ are suitable parameterised functions, whose structure remains equal in all rules and only the parameters vary. The parametrisation exploited here has the affine form:

$$
y_{i}=a_{i}^{T} x+b_{i},
$$

where $a_{i}$ is a parameter vector and $b_{i}$ is a scalar offset. This description is known as affine TS model. The antecedent of each rule defines a (fuzzy) validity region for the corresponding affine consequent model. These descriptions are considered here due to their interesting approximation properties [11]. Note that the state-vector $x$ in (3) can be designed to obtain a dynamic model, as remarked in the following.

Before the output can be inferred, the degree of fulfilment of the antecedent denoted by $\lambda_{i}(x)$ has to be computed. For rules with multivariate antecedent fuzzy sets given by (2), the degree of fulfilment is simply $\lambda_{i}=\mu_{A_{i}}(x)$. The TS model reduces the inference process to a simple algebraic expression, via e.g. the fuzzy-mean defuzzification formula:

$$
y=\frac{\sum_{i=1}^{K} \lambda_{i}(x) y_{i}}{\sum_{i=1}^{K} \lambda_{i}(x)}
$$

where the membership degrees $\lambda_{i}$ are modelled as exponential functions.

In order to introduce dynamics in (2), the consequents are linear ARX models, where $n$ is the order of the dynamic system, $x(k)=[y(k-1), \cdots, y(k-n), u(k-1), \cdots, u(k-$ $n)]^{T}$, and $a_{i}=\left[\alpha_{1}^{(i)}, \cdots, \alpha_{n}^{(i)}, \delta_{1}^{(i)}, \cdots, \delta_{n}^{(i)}\right]$. Finally, with reference to the structure (4), a procedure proposed by the authors for the estimation of both the parameters $a_{i}, b_{i}$, and the order $n$ is exploited, as described in [12]. Moreover, the estimation of the membership degrees $\lambda_{i}$ of (4) used for the aggregation of the local affine submodels (3) was obtained via a data clustering method available in the literature [11].

Many clustering algorithms were proposed, see e.g. [11]. In particular, the algorithm exploited in this work is based on the optimisation of the basic c-means objective function and known as fuzzy c-means clustering algorithm [11]. Note that the clustering method is a tool exploited by this study, since the identification scheme proposed in [12] requires that the clustered data fit linear relations. However, the clustering algorithm recalled above is exploited here since it can be easily integrated with the identification approach proposed in [12], and already available as software program [11].
In general there are several methods for estimating the consequent parameters $a_{i}$ and $b_{i}$ in (3). It is assumed that errors are present in both the regressor and the regressand, as described by the relation (5) [12]:

$$
\left\{\begin{array}{l}
u(k)=u^{*}(k)+\tilde{u}(k) \\
y(k)=y^{*}(k)+\tilde{y}(k)
\end{array}\right.
$$

where $u^{*}(k)$ and $y^{*}(k)$ are the unknown noise-free data, the noise terms $\tilde{u}(k)$ and $\tilde{y}(k)$ are independent of every other term, whilst only $u(k)$ and $y(k)$ are measured. Therefore, the structure of the local affine submodel (3) in the $i$-th cluster with $i=1, \cdots, K$, and $K$ clusters, is solved in the mathematical framework of the so-called noise-rejection problem [12]. This approach can be interpreted as the minimisation of the prediction error of the local affine submodels, solved as a set of $K$ independent problems, as proposed in [12]. This scheme, which is usually preferred when the TS model is used as predictor, computes the consequent parameters by the socalled Frisch scheme [12].

It is worth observing that, when fuzzy identification via data clustering is considered, an important point concerns the determination of the optimal number of clusters $K$. When clustering real data without any a priori information about the data structure, a preliminary assumption of the number $K$ of underlying clusters of the data is required. The selected clustering algorithm then searches for $K$ clusters, regardless of whether they are really present in the data or not. The optimisation issue was investigated in [12].

\section{Fault Diagnosis Scheme Design}

It is assumed that the input-output measurements of the system under diagnosis are modelled by the relations (5). The prediction (or estimation error) $y(k)-\hat{y}(k)$ in faultfree conditions represents the model-reality mismatch, which accounts for process noise, parameter variations, disturbance, etc.

Neglecting the sensor dynamics, faults acting on the measured input and output signals $u(k)$ and $y(k)$ are modelled as:

$$
\left\{\begin{array}{l}
u(k)=u^{*}(k)+f_{u}(k) \\
y(k)=y^{*}(k)+f_{y}(k)
\end{array}\right.
$$

where the term $f_{u}(k)$ and $f_{y}(k)$ represent additive signals assuming values different from zero only in the presence of faults.

There are different approaches to generate the residual signals for fault diagnosis, see e.g. [9]. In this work the residuals are obtained from the comparison between the measured $y(k)$ and the estimated $\hat{y}(k)$ outputs:

$$
r(k)=\hat{y}(k)-y(k)
$$

After the residual generation, the residual evaluation is performed by a logic device that processes the redundant signals in order to detect when a fault occurs and to univocally identify the faulty actuator or sensor measurement.

The fault detection task is implemented here by using a simple thresholding logic. It is worth noting that the faults described in Section II-A may not be immediately detected, since 
the delay in the corresponding alarm normally depends on the fault mode. Therefore, suitable fault detection thresholds are fixed according to (8):

$$
\left\{\begin{array}{l}
\bar{r}-\delta \sigma_{r} \leq r(k) \leq \bar{r}+\delta \sigma_{r} \\
\text { in fault-free conditions } \\
r(k)<\bar{r}-\delta \sigma_{r} \text { or } r(k)>\bar{r}+\delta \sigma_{r} \\
\text { in faulty conditions }
\end{array}\right.
$$

In (8), the residual signal is represented by the random variable $r(k)$, whose sample mean and variance values are computed as follows:

$$
\left\{\begin{array}{l}
\bar{r}=\frac{1}{N} \sum_{k=1}^{N} r(k) \\
\sigma_{r}^{2}=\frac{1}{N} \sum_{k=1}^{N}[r(k)-\bar{r}]^{2}
\end{array}\right.
$$

$\bar{r}$ and $\sigma_{r}^{2}$ are the values for the sample mean and variance of the fault-free residual $r(k)$, respectively. $N$ is the number of samples of $r(k)$. The values $\bar{r}$ and $\sigma_{r}^{2}$ depend on the signal $r(k)$ statistics, usually unknown. $\delta$ represents a design parameter.

In order to separate the normal from the faulty case, the tolerance parameter $\delta$ in (8) is selected and properly tuned. Hence, the proper choice of this parameter $\delta$ leads to a good trade-off between the maximisation of the fault detection probability and the minimisation of the false alarm rate. This parameter $\delta$ could be fixed with empirical rules (normally $\delta \geq 2$ ) or, once the values of $\bar{r}$ and $\sigma_{r}^{2}$ are estimated from the $r(k)$ signal, using the 3-sigma rule. On the other hand, less conservative results are obtained with a procedure that determines via extensive simulations the optimal $\delta$ minimising the false alarm rate and maximising the detection/isolation probability. This tuning methodology was proposed by the authors in a different framework [15] and based on MonteCarlo extensive simulations that were able to provide less conservative results than classic robust approaches. This issue will be discussed in Section V.

Finally, regarding the fault isolation problem, a Generalised Observer Scheme (GOS) is exploited [9]. In particular, as shown in Section II-A, different faults $f_{u}(k)$ or $f_{y}(k)$ affect the input or output measurements of the wind turbine system. Therefore, to uniquely isolate a fault $f_{u}(k)$ concerning one of the input signals, under the assumption that the output measurements are fault-free, a bank of fuzzy estimators (4) is used. The number of these estimators is equal to the number of the faults $f_{u}(t)$ to be diagnosed. The $i$-th fuzzy estimator is driven by all but the $i$-th input measurement (or even more inputs, if required) and all outputs of the system. It generates a residual signal that is sensitive to all but the $i$-th input fault $f_{u}(k)$ (or even more inputs, if necessary). The derivation of these fuzzy estimators follows the procedure described in Section III. Moreover, when the $i$-th fuzzy estimator insensitive to the $i$-th input is designed, the output $y(k)$ and all but the $i$-th inputs $u_{i}(k)$ are exploited for the identification process, according to the fault sensitivity analysis addressed in Section II-A. On the other hand, to uniquely isolate a fault $f_{y}(t)$ affecting one of the output measurements, under the hypothesis that the input signals are fault-free, a bank of fuzzy estimators is used again.

\section{Simulation Results}

The proposed FDI methodology is applied to $N=440 \times 10^{3}$ data $u(k)$ and $y(k)$ acquired from the wind turbine benchmark with a sampling rate of $100 \mathrm{~Hz}$. This wind turbine simulator is available from the web site http://www.kk-electronic.com/ wind-turbine-control/ competition-on-fault-detection.aspx.

According to Sections III and IV, the Gustafson-Kessel (GK) clustering method with $K=4$ clusters and a number of shifts $n=3$ was used for the identification of the fuzzy estimator banks of Section IV. These optimal parameters $K=4$ and $n=3$ were obtained as described in [12]. After the data clustering, the parameters $a_{i}$ and $b_{i}(i=1, \cdots, K)$ of the fuzzy estimators of the banks were computed using the identification method presented in Section III. Moreover, the fuzzy membership degrees $\lambda_{i}$ required by the fuzzy estimators (4) have been modelled as Gaussian functions.

The reconstructed output $\hat{y}_{i}(k)$ for the FDI task has been generated by a bank of 5 Multiple-Input Single-Output (MISO) fuzzy estimators (4). According to Table II, this scheme leads to the diagnosis of the fault cases $1,2,3,4$, and 5 reported in Table I. On the other hand, with reference again to Table II, a bank of 4 MISO fuzzy estimators allowed to diagnose the fault cases $6,7,8$, and 9 of Table I.

For each fault case, by following the FMEA procedure described in Section II-A and Table II, the input and output measurements used for the design of the fuzzy estimator banks are reported in Table III.

TABLE III

INPUTS, OUTPUTS AND RMSE FOR THE DESIGNED FUZZY RESIDUAL GENERATORS.

\begin{tabular}{c|ccc}
\hline Fault & Inputs & Output & RMSE \\
\hline 1 & {$\left[\beta_{1 m_{1}}(t), \beta_{1 m_{2}}(t)\right]$} & $\omega_{g m_{2}}(t)$ & 0.0164 \\
2 & {$\left[\beta_{2 m_{2}}(t), \beta_{1 m_{2}}(t)\right]$} & $\omega_{g m_{2}}(k)$ & 0.0233 \\
3 & {$\left[\beta_{3 m_{1}}(t), \beta_{1 m_{2}}(t)\right]$} & $\omega_{g m_{2}}(t)$ & 0.0209 \\
4 & {$\left[\beta_{1 m_{2}}(t), \omega_{g m_{2}}(t)\right]$} & $\omega_{r m_{1}}(t)$ & 0.0195 \\
5 & {$\left[\beta_{1 m_{2}}(t), \omega_{g m_{2}}(t)\right]$} & $\omega_{r m_{2}}(t)$ & 0.0187 \\
6 & {$\left[\beta_{2 m_{1}}(t), \beta_{1 m_{2}}(t)\right]$} & $\omega_{g m_{2}}(t)$ & 0.0213 \\
7 & {$\left[\beta_{3 m_{2}}(t), \beta_{1 m_{2}}(t)\right]$} & $\omega_{g m_{2}}(t)$ & 0.0177 \\
8 & {$\left[\beta_{1 m_{2}}(t), P_{g m}(t)\right]$} & $\omega_{g m_{2}}(t)$ & 0.0218 \\
9 & {$\left[\beta_{1 m_{2}}(t), \omega_{g m_{1}}(t)\right]$} & $\omega_{g m_{2}}(t)$ & 0.0189 \\
\hline
\end{tabular}

The approximation capabilities of the fuzzy estimators are expressed in terms of RMSE computed for the residuals $y(k)-\hat{y}(k)$ in fault-free conditions summarised in Table III. In particular, these RMSE values show that the estimated fuzzy models approximate the process outputs quite accurately.

The rationale for the use of TS fuzzy models was highlighted in Section III, whilst their efficacy is analysed in the following. As an example, the measured and the reconstructed outputs are depicted in Figure 1. The black continuous line represents the measured signal $y(k)$, i.e. $\omega_{g m_{2}}$, whilst the dotted gray line depicts its reconstruction $\hat{y}(k)$, provided by the fuzzy TS model for $\omega_{g m_{2}}(t)$.

The simulations reported in the following were obtained by considering the case of the Fault 4 , i.e. $f_{u}(t)$ commencing at 


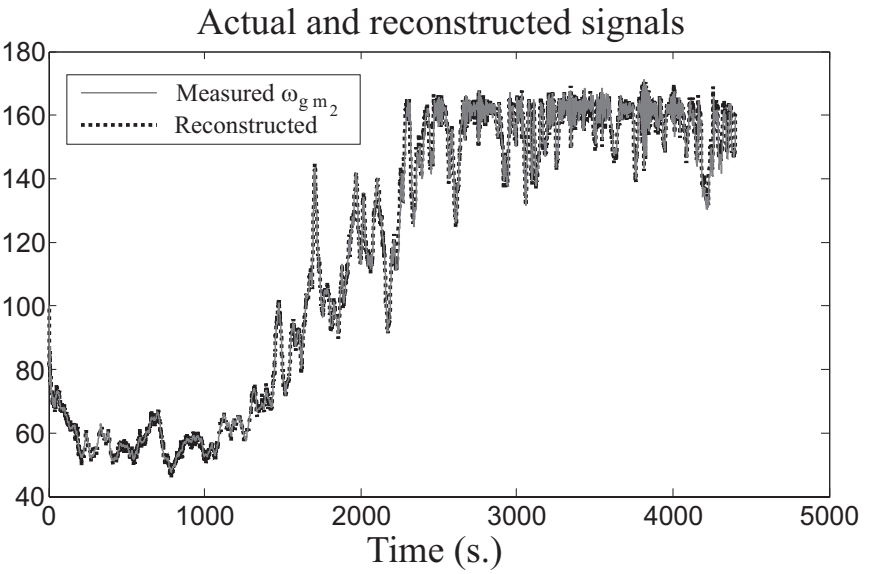

Fig. 1. Reconstructed $\hat{y}(k)$ and measured $y(k)$ output signals of the variable $\omega_{g} m_{2}$.

the instant $t=1500 \mathrm{~s}$. On the other hand, the effect of the Fault 8 , corresponding to $f_{y}(t)$ was also shown. This fault is active for $100 \mathrm{~s}$., between $3800 \mathrm{~s}$. and $3900 \mathrm{~s}$. The considered faults caused the change of the signals $u(t)$ and $y(t)$, as well as the residuals $r_{I_{i}}(t)$ generated by the fuzzy estimators (4). These residuals indicate the fault occurrence according to the logic (8), whether their values are lower or higher than the thresholds fixed in fault-free conditions. Fig. 2 represents the fault-free (grey continuous line) and the faulty (black dashed line) residual signals $r_{I_{i}}(t)$.

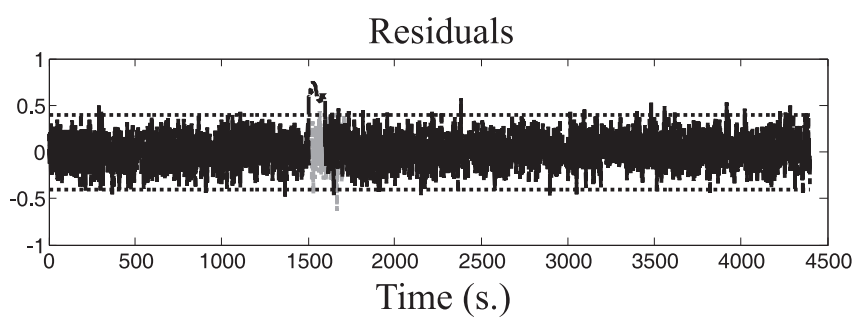

Fig. 2. Fault 4 residuals $r_{I_{i}}(t)$.

Fig. 2 depicts the fault detection thresholds (8) using dotted constant lines. Their values were properly settled as described in Section $\mathrm{V}-\mathrm{C}$, in order to minimise the false alarm and missed fault rates, while maximising the correct detection and isolation rates. In these conditions, the fault is correctly detected and isolated when the corresponding residual signal exceeds the thresholds, as shown in Fig. 2.

\section{A. Comparative Analysis}

This section provides comparative results with respect to the FDI schemes recalled in [7]. The first alternative approach considered here uses a Support Vector Machine based on a Gaussian kernel (GKSV). The second scheme consists of an Estimation Based (EB) solution, whilst the third method relies on Up-Down Counters (UDCs). The fourth approach combines Observer and Kalman Filter (COK) methods, and the fifth method relies on the General Fault Model (GFM) scheme, which is a method of automatic design.
For performance evaluation and comparison of the considered FDI schemes, some indices have been used, since already exploited by the authors e.g. in [15] for the reliability evaluation, and here computed on 1000 Monte-Carlo runs. These indices are defined as:

- False Alarm Rate $\left(r_{f a}\right)$ : the number of wrongly detected faults divided by total fault cases;

- Missed Fault Rate $\left(r_{m f}\right)$ : for each fault, the total number of undetected faults, divided by the total number of times that the fault case occurs;

- True Detection/Isolation Rate $\left(r_{t d i}\right)$ : for a particular fault case, the number of times it is correctly detected/isolated, divided by total number of times that the fault case occurs;

- Mean Detection/Isolation Delay $\left(\tau_{m d i}\right)$ : for a particular fault case, the average detection/isolation delay time.

These criteria are computed for the fault cases from 1 to 8 for the optimised FPRG scheme. The results of the remaining FDI strategies, which were not designed for the FDI of the fault 9, were simply inferred from [7] and transformed into values consistent with the considered performance criteria. Note also that [7] reported some statistics simply 0 or 1 for the specific FDI method and fault case. However, with respect to [7] Table IV summarises also the results obtained by considering the Fuzzy Predictors as Residual Generators (FPRG) presented in this work, and with an optimal choice of the threshold parameter $\delta$ in Eqs. (8) that leads to achieve optimal results.

TABLE IV

COMPARISON OF THE CONSIDERED FDI STRATEGIES.

\begin{tabular}{|c|c|cccccc|}
\hline Fault & Indices & GKSV & EB & UDC & COK & GFM & FPRG \\
\hline $\mathbf{1}$ & $r_{f a}$ & 0 & 0 & 0 & 0.890 & 0 & 0.001 \\
& $r_{m f}$ & 0 & 0.030 & 0 & 0 & 0 & 0.001 \\
& $r_{t d i}$ & 1 & 0.970 & 1 & 1 & 1 & 0.999 \\
& $\tau_{m d i}$ & $0.02 \mathrm{~s}$. & $0.02 \mathrm{~s}$. & $0.03 \mathrm{~s}$. & $10.32 \mathrm{~s}$. & $0.04 \mathrm{~s}$. & $0.02 \mathrm{~s}$. \\
\hline $\mathbf{2}$ & $r_{f a}$ & 0.560 & 0.220 & 0 & 0.970 & 0.308 & 0.001 \\
& $r_{m f}$ & 0 & 0.560 & 0.670 & 0 & 0 & 0.003 \\
& $r_{t d i}$ & 1 & 0.440 & 0.330 & 1 & 1 & 0.997 \\
& $\tau_{m d i}$ & $47.24 \mathrm{~s}$. & $44.65 \mathrm{~s}$. & $69.12 \mathrm{~s}$. & $19.24 \mathrm{~s}$. & $13.70 \mathrm{~s}$. & $0.08 \mathrm{~s}$. \\
\hline $\mathbf{3}$ & $r_{f a}$ & 0 & 0.040 & 0 & 0.014 & 0.016 & 0.003 \\
& $r_{m f}$ & 0 & 0.030 & 0.030 & 0 & 0 & 0.008 \\
& $r_{t d i}$ & 1 & 0.970 & 0.970 & 1 & 1 & 0.992 \\
& $\tau_{m d i}$ & $0.02 \mathrm{~s}$. & $0.54 \mathrm{~s}$. & $0.04 \mathrm{~s}$. & $10.35 \mathrm{~s}$. & $0.05 \mathrm{~s}$. & $0.02 \mathrm{~s}$. \\
\hline $\mathbf{4}$ & $r_{f a}$ & 0 & 0 & 0.010 & 0.023 & 0.033 & 0.004 \\
& $r_{m f}$ & 0 & 0 & 0 & 0 & 0 & 0.004 \\
& $r_{t d i}$ & 1 & 1 & 1 & 1 & 1 & 0.996 \\
& $\tau_{m d i}$ & $0.11 \mathrm{~s}$. & $0.33 \mathrm{~s}$. & $0.02 \mathrm{~s}$. & $0.18 \mathrm{~s}$. & $0.10 \mathrm{~s}$. & $0.02 \mathrm{~s}$. \\
\hline $\mathbf{5}$ & $r_{f a}$ & 0 & 0.117 & 0.075 & 0.026 & 0.024 & 0.002 \\
& $r_{m f}$ & 0.030 & 0 & 0 & 0.014 & 0 & 0.003 \\
& $r_{t d i}$ & 0.070 & 1 & 1 & 0.986 & 1 & 0.997 \\
& $\tau_{m d i}$ & $25.90 \mathrm{~s}$. & $0.01 \mathrm{~s}$. & $2.96 \mathrm{~s}$. & $31.32 \mathrm{~s}$. & $9.49 \mathrm{~s}$. & $0.03 \mathrm{~s}$. \\
\hline $\mathbf{6}$ & $r_{f a}$ & 1 & 0.020 & 0.022 & 0.003 & 0.036 & 0.042 \\
& $r_{m f}$ & 1 & 0 & 0 & 0 & 0 & 0.033 \\
& $r_{t d i}$ & 0 & 1 & 1 & 1 & 1 & 0.967 \\
& $\tau_{m d i}$ & N/A & $11.31 \mathrm{~s}$. & $11.81 \mathrm{~s}$. & $23.80 \mathrm{~s}$. & $12.52 \mathrm{~s}$. & $3.03 \mathrm{~s}$. \\
\hline $\mathbf{7}$ & $r_{f a}$ & 0 & 0.018 & 0.020 & 0 & 0.012 & 0.047 \\
& $r_{m f}$ & 1 & 0 & 0 & 0 & 0 & 0.023 \\
& $r_{t d i}$ & 0 & 1 & 1 & 1 & 1 & 0.977 \\
& $\tau_{m d i}$ & N/A & $26.07 \mathrm{~s}$. & $12.93 \mathrm{~s}$. & $34.00 \mathrm{~s}$. & $31.70 \mathrm{~s}$. & $5.07 \mathrm{~s}$. \\
\hline $\mathbf{8}$ & $r_{f a}$ & 0 & 0 & 0 & 0 & 0 & 0.003 \\
& $r_{m f}$ & 0.970 & 0.970 & 0.970 & 0.970 & 0.970 & 0.002 \\
& $r_{t d i}$ & 0.030 & 0.030 & 0.030 & 0.030 & 0.030 & 0.998 \\
& $\tau_{m d i}$ & $0.01 \mathrm{~s}$. & $0.01 \mathrm{~s}$. & $0.02 \mathrm{~s}$. & $0.01 \mathrm{~s}$. & $7.92 \mathrm{~s}$. & $0.05 \mathrm{~s}$. \\
\hline
\end{tabular}

It is worth noting that, as remarked in [7] the detection of fault 8 is quite difficult, due to the fault nature, as shown by the missed detection statistics for all schemes. Moreover, most 
of the considered solutions are able to diagnose the fault 2, even if, as described in [8], active fault diagnosis approaches can work better, as shown for the results in Section V-B.

The GKSV solution is able to diagnose the sensor fault cases from 1 to 5 , and for most of them without any false alarm rates. This scheme provided good robustness toward the changed point of operation of the faults. The EB scheme showed good detection delays for the original test data, but it is slower when the fault location changes. Large false alarm rates are present for some faults, which could be improved with a different choice of the design parameters, as shown for the FPRG approach in Section V-C. The UDC approach is able to diagnose almost all faults, which are detected and isolated quickly, but with relevant false alarm rates. On the other hand, the COK solution worked well for most of the faults, even if quite slowly, and with some relevant false alarm rates. The GFM method is able to diagnose all faults (except for the case 8) quite slowly and with some relevant false alarm rates. Finally, regarding the proposed FPRG method, it seems to work relatively better than the others, even if optimisation stages are required, for example, for the optimal FDI threshold selection. For this method, in general, which is tested also for fault 9 , the detection rates are bigger than $83 \%$, with false alarm and missed fault rates lower than $14 \%$. The issue of the optimal threshold selection will be analysed in Section V-C, since this key point was not considered in [7].

\section{B. Active Fault Diagnosis Comparison}

As already remarked for Table IV, the FDI schemes presented in [7] were not evaluated for the fault case 9. Therefore, this section provides further comparative results with respect to different fault diagnosis schemes addressed in [8], but proposed for active fault tolerant control, and applied to the same benchmark, where the fault case 9 is also considered. In more detail, the properties of the datadriven FDI method suggested in this paper have been analysed with respect to alternative active fault diagnosis approaches, in particular relying on Adaptive Filters obtained via the NonLinear Geometric Approach (NLGA-AF) and described in [8], the Sliding Mode Observer (SMO) [16], and Neural Network (NN) estimators [17]. These schemes were already designed for this benchmark, as described in [8].

It is worth noting that, in particular with reference to offshore installations, where the $\mathrm{O} \& \mathrm{M}$ costs can be very expensive, a proper fault detection scheme should be able to highlight anomalous working conditions in their earlier occurrence. In particular, a fault accommodation schemes, even if relying on adaptive solutions, should be able to maintain a 'graceful' degradation of the working conditions, as soon as the $\mathrm{O} \& \mathrm{M}$ operations are performed.

In order to provide a brief but clear insight into the above mentioned techniques, the comparison has been performed in the same previous working conditions, and based on the performance indices. It is worth recalling the main features of the alternative fault diagnosis schemes implemented via the NLGA-AF, SMO and the NN estimator. In particular, the NLGA-AF tries to decouple the disturbance effects in an analytical way, and exploiting the NLGA scheme. The SMO strategy is able to decouple the model-reality uncertainty via the sliding motion, whilst the NN estimators were designed to passively tolerate disturbance and modelling errors. Table V shows the performances of the different FDI schemes.

TABLE V

COMPARISON OF THE RESULTS SHOWN IN [8].

\begin{tabular}{|c|c|c|c|c|c|}
\hline Fault & Indices & NLGA-AF & SMO & $\mathbf{N N}$ & FPRG \\
\hline \multirow[t]{4}{*}{1} & $r_{f a}$ & 0.002 & 0.002 & 0.012 & 0.001 \\
\hline & $r_{m f}$ & 0.022 & 0.014 & 0.213 & 0.001 \\
\hline & $r_{t d i}$ & 0.978 & 0.986 & 0.787 & 0.999 \\
\hline & $\tau_{m d i}$ & $0.03 \mathrm{~s}$. & $0.04 \mathrm{~s}$. & $0.16 \mathrm{~s}$ & $0.02 \mathrm{~s}$. \\
\hline \multirow[t]{4}{*}{2} & $r_{f a}$ & 0.277 & 0.267 & 0.201 & 0.001 \\
\hline & $r_{m f}$ & 0.334 & 0.233 & 0.422 & 0.003 \\
\hline & $r_{t d i}$ & 0.666 & 0.767 & 0.578 & 0.997 \\
\hline & $\tau_{m d i}$ & $0.23 \mathrm{~s}$. & $0.55 \mathrm{~s}$. & $79.13 \mathrm{~s}$. & $0.08 \mathrm{~s}$. \\
\hline \multirow[t]{4}{*}{3} & $r_{f a}$ & 0.024 & 0.201 & 0.333 & 0.003 \\
\hline & $r_{m f}$ & 0.016 & 0.102 & 0.441 & 0.008 \\
\hline & $r_{t d i}$ & 0.984 & 0.898 & 0.559 & 0.992 \\
\hline & $\tau_{m d i}$ & $0.05 \mathrm{~s}$. & $0.64 \mathrm{~s}$. & $9.25 \mathrm{~s}$ & $0.02 \mathrm{~s}$ \\
\hline \multirow[t]{4}{*}{4} & $r_{f a}$ & 0.007 & 0.005 & 0.133 & 0.004 \\
\hline & $r_{m f}$ & 0.006 & 0.006 & 0.243 & 0.004 \\
\hline & $r_{t d i}$ & 0.974 & 0.994 & 0.757 & 0.996 \\
\hline & $\tau_{m d i}$ & $0.16 \mathrm{~s}$. & $0.34 \mathrm{~s}$. & $8.85 \mathrm{~s}$ & $0.02 \mathrm{~s}$ \\
\hline \multirow[t]{4}{*}{5} & $r_{f a}$ & 0.087 & 0.016 & 0.244 & 0.002 \\
\hline & $r_{m f}$ & 0.114 & 0.018 & 0.354 & 0.003 \\
\hline & $r_{t d i}$ & 0.886 & 0.982 & 0.646 & 0.997 \\
\hline & $\tau_{m d i}$ & $0.55 \mathrm{~s}$. & $0.07 \mathrm{~s}$ & $8.84 \mathrm{~s}$ & $0.03 \mathrm{~s}$ \\
\hline \multirow[t]{4}{*}{6} & $r_{f a}$ & 0.087 & 0.093 & 0.334 & 0.042 \\
\hline & $r_{m f}$ & 0.187 & 0.214 & 0.257 & 0.033 \\
\hline & $r_{t d i}$ & 0.813 & 0.786 & 0.743 & 0.967 \\
\hline & $\tau_{m d i}$ & $0.95 \mathrm{~s}$. & $0.37 \mathrm{~s}$. & $32.94 \mathrm{~s}$. & $3.03 \mathrm{~s}$. \\
\hline \multirow[t]{4}{*}{7} & $r_{f a}$ & 0.019 & 0.014 & 0.334 & 0.047 \\
\hline & $r_{m f}$ & 0.021 & 0.025 & 0.421 & 0.023 \\
\hline & $r_{t d i}$ & 0.979 & 0.975 & 0.579 & 0.977 \\
\hline & $\tau_{m d i}$ & $0.05 \mathrm{~s}$ & $0.07 \mathrm{~s}$. & $23.93 \mathrm{~s}$ & $5.07 \mathrm{~s}$. \\
\hline \multirow[t]{4}{*}{8} & $r_{f a}$ & 0.014 & 0.035 & 0.344 & 0.003 \\
\hline & $r_{m f}$ & 0.017 & 0.041 & 0.401 & 0.002 \\
\hline & $r_{t d i}$ & 0.983 & 0.959 & 0.599 & 0.998 \\
\hline & $\tau_{m d i}$ & $0.09 \mathrm{~s}$. & $0.08 \mathrm{~s}$. & $14.19 \mathrm{~s}$. & $0.05 \mathrm{~s}$ \\
\hline \multirow[t]{4}{*}{9} & $r_{f a}$ & 0.278 & 0.379 & 0.594 & 0.134 \\
\hline & $r_{m f}$ & 0.296 & 0.234 & 0.437 & 0.165 \\
\hline & $r_{t d i}$ & 0.704 & 0.766 & 0.563 & 0.835 \\
\hline & $\tau_{m d i}$ & $0.25 \mathrm{~s}$. & $0.27 \mathrm{~s}$. & $52.94 \mathrm{~s}$. & $0.30 \mathrm{~s}$. \\
\hline
\end{tabular}

The comparison of Table $\mathrm{V}$ highlights that the scheme using NLGA-AF and SMO allows to achieve good performances, even if the SMO can increase the computational time.

Few further comments can be drawn here. When the modelling of the dynamic system can be perfectly obtained, in general model-based strategies are preferred. On the other hand, when modelling errors and uncertainty are present, alternative estimation schemes relying on adaptation mechanisms, or passive approaches, such as the FPRG, have shown interesting robustness properties in the presence of unmodelled disturbance, modelling mismatch, and measurement errors. With reference to the $\mathrm{NN}$ estimator, in the case of a system with modelling errors, the off-line learning can lead to poor results. Other explicit disturbance decoupling techniques such as the NLGA-AF can take advantage of their robustness capabilities, but with quite complicated and not straightforward design procedures. The NN-based scheme relies on the learning accumulated from off-line simulations, but the training stage can be computationally heavy.

\section{Performance Evaluation}

This section reports further simulations regarding the performance evaluation of the developed FDI scheme with respect 
to modelling errors and measurement uncertainty.

With reference to the proposed fault diagnosis approach that exploits identified TS models from data sequences, it is worth noting that the authors did not try to improve the robustness of the identified TS models. Section V highlighted the capabilities of the identified fuzzy TS models used for FDI with the methodology recalled in Section III. This approach showed interesting approximation properties when applied also to real data. Therefore, the scheme seems e.g. similar to [18]. Another important issue concerns the analysis addressed in this section, which was applied to the determination of the FDI threshold values, as described in [15]. On the other hand, the use of the FMEA approach enhances the fault isolation task, thus obviating the need of unknown input or disturbance decoupling designs, as proposed e.g. in [19], [20]. Note also that, with respect $e$.g. to set-membership approaches described in [18], the strategy proposed in this paper can be considered as belonging to the so-called passive approaches, since the robustness is achieved at the decision-making stage by propagating the uncertainty to the residuals via adapted thresholds. On the other hand, among the active schemes, e.g. the works [19], [20] deal with the problem of designing robust FDI observers for nonlinear systems. In these cases, an unknown input observer is designed, which can tolerate a degree of model uncertainty, and hence increases the reliability of fault diagnosis. Moreover, whilst the methodology proposed in this paper enhances both the identification procedure and the fault isolation scheme design, the works [19], [20] address alternative structure and analytical UIO design procedure for nonlinear models. On the other hand, with reference to modelfree approaches, in general, they do not allow calculating the uncertainty of these estimated descriptions, which are necessary to apply them in robust fault detection schemes. To tackle these limitations, GMDH neural networks can be applied, as described e.g. in [21], [22]. The behaviour of each partial model in the GMDH neural network reflects that of the identified system. Therefore, with respect again to [21], [22], the robustness of the approach proposed in this work is achieved via adapted thresholds. However, the use of the identification approach recalled in Section III allows to calculate the model uncertainty, which then can be exploited for achieving robust fault detection features, as shown in the following.

After there remarks, Table VI summarises the results obtained by considering the fuzzy predictors with a choice of the parameter $\delta$ in (8) that leads to optimal performance.

The tuning of the thresholds in (8) consists of an optimal choice of the parameter $\delta$ in the residual evaluation for obtaining almost zero false alarm rates, whilst the performances were evaluated by simulating a number of experiments of a Monte Carlo analysis [15].

The tuning strategy regards mainly the choice of the parameter used for the residual evaluation in (8). In particular, for each possible value of $\delta$ varying in a range of $\left(\sigma_{r}, 5 \sigma_{r}\right)$, where $\sigma_{r}$ represents the standard deviation of the fault-free residual $r(k)$, the Monte Carlo analysis determines the performance indices described above. Thus, by varying the value of $\delta$ with a simple and straightforward exhaustive local search algorithm,
TABLE VI

OPTIMISATION RESULTS.

\begin{tabular}{|c|ccccc|}
\hline Fault & $r_{f a}$ & $r_{m f}$ & $r_{t d i}$ & $\tau_{m d i}$ & $\delta$ \\
\hline $\mathbf{1}$ & 0.001 & 0.001 & 0.999 & $0.02 \mathrm{~s}$. & 3.9 \\
\hline $\mathbf{2}$ & 0.001 & 0.003 & 0.997 & $0.08 \mathrm{~s}$. & 4.1 \\
\hline $\mathbf{3}$ & 0.003 & 0.008 & 0.992 & $0.02 \mathrm{~s}$. & 3.9 \\
\hline $\mathbf{4}$ & 0.004 & 0.004 & 0.996 & $0.02 \mathrm{~s}$. & 4.3 \\
\hline $\mathbf{5}$ & 0.002 & 0.003 & 0.997 & $0.03 \mathrm{~s}$. & 3.5 \\
\hline $\mathbf{6}$ & 0.042 & 0.033 & 0.967 & $3.03 \mathrm{~s}$. & 4.6 \\
\hline $\mathbf{7}$ & 0.047 & 0.023 & 0.977 & $5.07 \mathrm{~s}$. & 4.5 \\
\hline $\mathbf{8}$ & 0.003 & 0.002 & 0.998 & $0.05 \mathrm{~s}$. & 3.7 \\
\hline $\mathbf{9}$ & 0.134 & 0.165 & 0.835 & $43.03 \mathrm{~s}$. & 2.8 \\
\hline
\end{tabular}

the threshold levels and the indices vary accordingly [15]. This optimisation and tuning resulted in minimising the $r_{f a}, r_{m f}$, and $r_{t d i}$. It is worth noting that the proposed tuning tool can obviate the need of other analytical designs, which can be exploited to improve the robustness of the FDI method [9]. To this aim, the authors did not try to improve the robustness of the identified fuzzy models since they were already suitable for the considered application.

Finally, Table VI shows that the proper selection of $\delta$ in (8) leads to achieve false alarm and missed fault rates less than $13 \%$, and detection/isolation rates larger than $83 \%$, with minimal detection/isolation delay times. The results demonstrate also that the Monte-Carlo analysis is an effective tool for tuning and testing the suggested FDI method. Moreover, in presence of uncertainty and modelling errors, this simulation tool seems to facilitate the assessment of the reliability and the robustness of the developed FDI scheme for application to real cases.

\section{CONCLUSION}

This paper proposed a methodology for the fault detection and isolation of a wind turbine system using fuzzy models identified from input-output measurements. The process under investigation is nonlinear and its measurements are noisy. The residual generators designed for diagnostic purpose has the form of Takagi-Sugeno models. These fuzzy models were derived using fuzzy clustering and dynamic system identification techniques. The effectiveness of the proposed approach was tested on the data acquired from the simulated wind turbine benchmark. The achieved results showed that the detection and isolation of the faults affecting sensors, component and actuators of the process under diagnosis was possible. Future investigations will address the application of the diagnosis strategy to real wind turbine installations.

\section{REFERENCES}

[1] A. Stefani, A. Bellini, and F. Filippetti, "Diagnosis of Induction Machines' Rotor Faults in Time-Varying Conditions," IEEE Trans. Ind. Electron., vol. 56, no. 11, pp. 4548-4556, 2009.

[2] X. Gong and W. Qiao, "Bearing Fault Diagnosis for Direct-Drive Wind Turbines via Current-Demodulated Signals," IEEE Trans. Ind. Electron., vol. 60, no. 8, pp. 3419-3428, 2013.

[3] N. M. A. Freire, J. O. Estima, and A. J. Marques Cardoso, "Open-Circuit Fault Diagnosis in PMSG Drives for Wind Turbine Applications," IEEE Trans. Ind. Electron., vol. 60, no. 9, pp. 3957-3967, 2013. 
[4] M. A. Parker, H. N. Chong, and L. Ran, "Fault-Tolerant Control for a Modular Generator-Converter Scheme for Direct-Drive Wind Turbines," IEEE Trans. Ind. Electron., vol. 58, no. 1, pp. 305-315, 2011.

[5] F. Rachidi, M. Rubinstein, J. Montanya, J. L. Bermudez, R. Rodriguez Sola, G. Sola, and N. Korovkin, "A Review of Current Issues in Lightning Protection of New-Generation Wind-Turbine Blades," IEEE Trans. Ind. Electron., vol. 55, no. 6, pp. 2489-2496, 2008.

[6] K. Rothenhagen and F. W. Fuchs, "Doubly Fed Induction Generator Model-Based Sensor Fault Detection and Control Loop Reconfiguration," IEEE Trans. Ind. Electron., vol. 56, no. 10, pp. 4229-4238, 2009.

[7] P. F. Odgaard, J. Stoustrup, and M. Kinnaert, "Fault-Tolerant Control of Wind Turbines: A Benchmark Model," IEEE Trans. Contr. Syst. Technol., vol. 21, no. 4, pp. 1168-1182, 2013.

[8] S. Simani and P. Castaldi, "Data-Driven and Adaptive Control Applications to a Wind Turbine Benchmark Model," Control Eng. Prac., vol. 21, no. 12 , pp. $1678-1693,2013$.

[9] J. Chen and R. J. Patton, Robust Model-Based Fault Diagnosis for Dynamic Systems. Boston, MA, USA: Kluwer Academic Publishers, 1999.

[10] S. Simani, C. Fantuzzi, and R. J. Patton, Model-based fault diagnosis in dynamic systems using identification techniques, 1st ed., ser. Advances in Industrial Control. London, UK: Springer-Verlag, 2003.

[11] R. Babuška, Fuzzy Modeling for Control. Boston, USA: Kluwer Academic Publishers, 1998.

[12] S. Simani, C. Fantuzzi, R. Rovatti, and S. Beghelli, "Parameter identification for piecewise linear fuzzy models in noisy environment," International Journal of Approximate Reasoning, vol. 1, no. 22, pp. 149-167, 1999.

[13] S. Simani and M. Bonfè, "Fuzzy modelling and control of the air system of a real diesel engine," Advances in Fuzzy Systems, vol. 2009, pp. 1-14, 2009.

[14] S. Simani and P. Castaldi, "Active Actuator Fault Tolerant Control of a Wind Turbine Benchmark Model," Int. J. Robust Nonlin., vol. 24, no. 8-9, pp. 1283-1303, 2014.

[15] R. J. Patton, F. J. Uppal, S. Simani, and B. Polle, "Robust FDI applied to thruster faults of a satellite system," Control Eng. Prac., vol. 18, no. 9, pp. 1093-1109, 2010.

[16] C. Edwards, S. K. Spurgeon, and R. J. Patton, "Sliding mode observers for fault detection and isolation," Automatica, vol. 36, no. 1, pp. 541$553,2000$.

[17] J. Korbicz, J. M. Koscielny, Z. Kowalczuk, and W. Cholewa, Eds., Fault Diagnosis: Models, Artificial Intelligence, Applications, 1st ed. London, UK: Springer-Verlag, 2004

[18] V. Puig, "Fault Diagnosis and Fault Tolerant Control using SetMembership Approaches: Application to Real Case Studies," Int. J. Appl. Math. Comput. Sci., vol. 20, no. 4, pp. 619-635, 2010.

[19] M. Witczak and P. Pretkia, "Design of an extended unknown input observer with stochastic robustness techniques and evolutionary algorithms," International Journal of Control, vol. 80, no. 5, pp. 749-762, 2007.

[20] J. Korbicz, M. Witczak, and V. Puig, "LMI-based strategies for designing observers and unknown input observers for non-linear discretetime systems," Bulletin of the Polish Academy of Sciences: Techinical Sciences, vol. 55, no. 1, pp. 31-42, 2007.

[21] M. Mrugalski, "An unscented Kalman filter in designing dynamic GMDH neural networks for robust fault detection," Int. J. Appl. Math. Comput. Sci., vol. 23, no. 1, pp. 157-169, 2013

[22] V. Puig, M. Witczak, F. Nejjari, J. Quevedo, and J. Korbicz, "A GMDH neural network-based approach to passive robust fault detection using a constraint satisfaction backward test," Engineering Applications of Artificial Intelligence, vol. 20, no. 7, pp. 886-897, 2007.

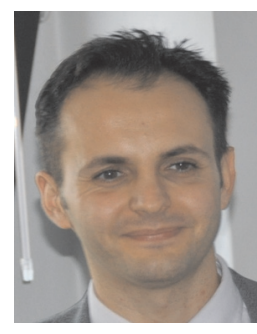

Silvio Simani received the Laurea degree (cum laude) in Electronic Engineering in 1996, and the $\mathrm{Ph} . \mathrm{D}$. in Information Science (Automatic Control) in 2000. Since 2006 he has been an IEEE Senior Member, and from 2000 a member of the SAFEPROCESS Technical Committee. Since 2002 he has been an Assistant Professor at the Department of Engineering of the University of Ferrara. His research interests include fault diagnosis, fault tolerant control, and system identification, on which he published about 160 refereed journal and conference papers, as well as three books and chapters.

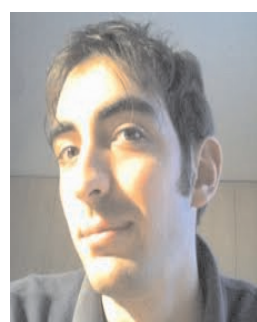

Saverio Farsoni was born in Mirandola (MO, Italy) in 1987. In $2012 \mathrm{He}$ graduated (cum laude) in Informatics and Automation Engineering at the University of Ferrara with a M. Sc. thesis on simulations in bio-medical environments. Since 2013 he has been $\mathrm{PhD}$ student in Engineering Science and, together with his supervisor, Dr. Simani, he works on control systems, fuzzy logic, modelling and identification problems. In particular, his researches deal with fault diagnosis and fault tolerant control for eolic plants, and he published some conference papers about these issues.

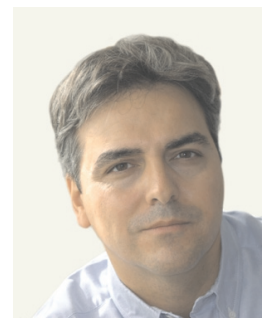

Paolo Castaldi received the Laurea degree (cum laude) in Electronic Engineering in 1990 and the $\mathrm{Ph} . \mathrm{D}$ degree in System Engineering in 1994. Since 1995 he has been an Assistant Professor at the Department of Electrical, Electronic and Information Engineering of the University of Bologna. Since 2009 he has been a member of the IFAC Technical Committee on Aerospace. He has published about 100 refereed journal and conference papers, several book's chapters, and 1 monograph. His research interests include fault diagnosis and fault tolerant control, adaptive filtering, system identification, and their applications to aerospace and mechanical systems. 